\title{
EVALUATION OF STANDARD QUESTIONNAIRE TO ASSESS CHRONIC ALCOHOLISM
}

\author{
Chelladurai Kandaswamy¹, Panimathi Rengaramanujam²
}

${ }_{1}^{1}$ Associate Professor, Department of Biochemistry, Madras Medical College, Chennai, Tamilnadu, India.

2Professor, Department of Biochemistry, Madras Medical College, Chennai, Tamilnadu, India.

\begin{abstract}
BACKGROUND
ABSTRACT

Off all the drugs, alcohol is the only drug whose self-intoxication is socially acceptable. Over the past years increasing percentage of young people have started drinking alcoholic beverages and their alcohol consumption has increased in quantity and frequency and the age at which drinking starts has declined. This situation is disturbing because young people may run a greater risk of alcohol related problems in their later life and also in the short term, there may be increase in road accidents. According to current concept, alcoholism is considered a disease and alcohol a disease causing agent. Alcohol screening is recommended as a component of routine preventive care for several reasons. The combined prevalence of at-risk drinking, alcohol abuse and alcohol dependence approaches $20 \%$ in any practice setting. Early identification and management of such drinking can avert more serious and irreversible health and social consequences. In most health care settings, the best source of information for screening is the history. Self-reports of drinking usually match or exceed report by family members. We wanted to apply and ask the standard questionnaire to persons who take alcohol, in any form, regularly and also in excess quantity and to measure serum GGT (Gamma Glutamyl Transferase/Transpeptidase) in their plasma, analyse it statistically and thereby evaluate the strength of the questionnaire.
\end{abstract}

\section{METHODS}

The study population consists of 50 persons who take alcohol regularly in excess of AUDIT score of less than 8 and 50 persons who take alcohol with AUDIT score of 8 and more. Apart from AUDIT, CAGE questionnaire was also applied. Serum Gamma Glutamyl Transferase was measured by manual method in both the groups and the results were analysed by the Mann-Whitney test.

\section{RESULTS}

The mean value of serum GGT was significantly elevated in cases who have AUDIT* score of more than 8 with a 'p' value at 0.001 of significance $(<0.05$ considered statistically significant).

\section{CONCLUSIONS}

The present study supports the hypothesis that excessive alcohol drinking increases the plasma GGT. Self-reporting of alcohol drinking under-estimates the true risk. Thus, the use of GGT- the biological marker of alcohol drinking is a helpful tool for risk assessment. Excessive intake of alcohol and raised GGT levels can go hand-in hand and a better understanding of the mechanism between alcohol drinking, biological markers, and the importance of AUDIT questionnaire is the base line tool for screening people for their drinking behaviour. Usually the AUDIT scoring method is followed in many studies to estimate the prevalence of drinking in a community. This study attempts to compare the questionnaire and the biological marker-GGT. The combination of questionnaire and the marker is equally good as the questionnaire. GGT tests the tissue level while the questionnaire reveals the social behaviour of alcohol consumption. A larger study may throw light upon bias if it exists.

\section{KEY WORDS}

Questionnaire, Alcohol, GGT, CAGE (Cut-Down, Annoyed, Guilty, Eye Opener), AUDIT (Alcohol Use Disorder Identification Test)

HOW TO CITE THIS ARTICLE: Kandaswamy C, Rengaramanujam P. Evaluation of standard questionnaire to assess chronic alcoholism. J. Evolution Med. Dent. Sci. 2019;8(24):1896-1899, DOI: 10.14260/jemds/2019/417

\section{BACKGROUND}

According to current concept, alcoholism is considered a disease and alcohol a disease causing agent. The health problem for which alcohol is responsible is only part of the total social damage it causes. ${ }^{1}$

An estimated $4.5 \%$ of the global burden of disease as measured in disability-adjusted life years is caused by

'Financial or Other Competing Interest': None.

Submission 23-04-2019, Peer Review 03-06-2019,

Acceptance 10-06-2019, Published 17-06-2019.

Corresponding Author:

Dr. Panimathi Rengaramanujam,

Professor

Department of Biochemistry,

Madras Medical College, Chennai,

Tamilnadu, India.

E-mail: parimathi@hotmail.com

DOI: $10.14260 /$ jemds $/ 2019 / 417$

\section{(c) (1) $(9)$}

harmful use of alcohol. ${ }^{2}$ India, which was considered as one of the countries with tradition of abstinence, is a thing of past. Alcohol consumption has now become a social activity and is accepted as a casual behaviour. A nationwide survey on drug abuse showed that the prevalence of alcohol consumption was $21 \%$ among men and $2 \%$ among women in India. $^{3} \mathrm{~A}$ number of factors are associated at the individual and social levels to determine the magnitude and pattern of alcohol consumption and alcohol use disorders such as age, sex, familial factors, drinker's behaviour, alcohol exposure (Volume, patterns, and quality of alcohol consumed), economic development, culture, and existing alcohol-related policies.[4]

\section{Alcohol Screening}

Alcohol screening is recommended as a component of routine preventive care for several reasons. The combined prevalence of at-risk drinking, alcohol abuse and alcohol 
dependence approaches $20 \%$ in any practice setting. Early identification and management of such drinking can avert more serious and irreversible health and social consequences. In most health care settings the best source of information for screening is the history. Self-reports of drinking usually match or exceed report by family members. Several brief batteries of yes or no screening questions can identify most individuals with at-risk drinking and alcoholuse disorders. Such questions can be incorporated conversationally into routine histories. The CAGE and AUDIT questionnaires (detailed below) are used most often and are most accurate in adult men.

\section{Alcohol Screening Questionnaire}

A number of groups including the National Institute on Alcohol Abuse and Alcoholism (NIAAA), the US Preventive Task Force and The American Academy of Family Physicians reviewed the issue of screening and have recommended screening of adult and adolescent patients for problematic use of alcohol. The most effective method for detecting alcohol problems is to elicit an alcohol history by asking quantity and frequency questions in a routine manner and using a standardized screening questionnaire. Apart from routine screening the standardized questionnaire are CAGE, AUDIT (detailed below).

\section{CAGE; Cut-down, Annoyed, Guilty, Eye Opener} Questionnaire

1. Have you ever felt that you should cut-down your drinking?

2. Have people annoyed you by criticizing your drinking?

3. Have you ever felt bad or guilty about your drinking?

4. Have you ever taken a drink the first thing in the morning? (Eye-opener) to steady your nerves or get rid of a hang-over?

One 'yes' suggests a need for further assessment.

\section{Audit; Alcohol Use Disorder Identification Test} Questionnaire

1. How often do you have taken a drink containing alcohol?

2. How many drink containing alcohol, do you have on a typical day when you are drinking?

3. How often do you have more than 6 drink in one occasion?

4. How often during the last year have you found that you were not able to stop drinking once you have started?

5. How often during the last year you have failed to do what was normally expected of you because of drinking?

6. How often during the last year you needed the first drink in the morning to get yourself going after a heavy drinking?

7. How often during the last year you had a feeling of guilt or remorse after drinking?

8. How often during the last year you have been unable to remember what happened the night before you had been drinking?

9. Have you or someone else been injured as a result of your drinking?
10. Has a relative or a friend or a doctor or other health care worker been concerned about your drinking or suggested you to cut down?

\section{Scoring Audit}

Questions 1-8 are scored 0, 1, 2, 3 or 4. Questions 9 and 10 are scored 0,2 or 4 only-

\section{Question 1}

0 - Never

1 - Monthly or less

2-2-4 times a month

3 - 2-3 times a week

4 -More than 4 times a week

\section{Question 2}

0- 1-2 drink (1 drink $30 \mathrm{ml}$ approximately)

1- 3 or 4

2- 5 or 6

3- 7-9

4 more than 10 drink

\section{Question 3 to 8}

0 - Never.

1- Less than monthly.

2- Monthly.

3- Weekly.

4- Daily almost.

\section{Question 9 to 10 (2 Point or 4 Points Grading Only)}

0 -No

2-Yes but not last year

3-Yes, during last year.

The AUDIT focuses on social and behavioural aspect of alcohol problem and provides greater accuracy than do frequency questions, lab test or clinical detection.

\section{More than 8 Point}

Positive for an alcohol use-disorder (AUD) for both men and women. 5

\section{Abnormalities of Plasma Enzymes}

Plasma gamma glutamyltransferase: It is now more than 35 years since S. B. Rosalki first demonstrated the special sensitivity of the plasma gamma glutamyltransferase to excess alcohol intake. This sensitivity has repeatedly been confirmed and this remains the most valuable of all single bio-chemical measures for the detection of chronic excess alcohol intake. The enzyme originates in the liver where alcohol induces it's synthesis. This is an early effect of alcohol which precedes abnormalities of conventional liver function tests may occur independently of abnormal liver histology. In general GGT values are on an average 2 to 3 times higher than the upper reference limit in hospitalized alcoholics and oneand-a-half times higher in alcoholic out-patients. Higher values at equivalent levels of consumption have been observed in Asian Indians when compared to white Europians. The frequency of missed plasma GGT in alcoholics is greater with regular daily drinking than with bout drinking and increases with duration of drinking before sampling. ${ }^{6} \mathrm{At}$ times GGT may be the only abnormal liver function test on 
routine clinical examination, suggesting the presence of occult alcohol abuse. The enzyme can be used to monitor alcohol abstinence since it's activity increases upon a return to alcohol abuse. Abstinence results in a return to normal in 2-5 weeks. The drug that induces the synthesis of GGT are Phenytoin, Phenobarbitone, Rifampicin, Fibrates and OCP containing oestrogen. The increased level is not due to cell damage but to an increase in enzyme production within the cell and released during normal cell turn-over. ${ }^{6}$ Even among the group of healthy members who are at the risk of alcohol related medical and social problem the most reliable test is elevated levels of GGT.

\section{Aims and Objectives}

The objective is to apply and ask the standard questionnaire to persons who take alcohol, in any form, regularly and also excess in quantity and to measure serum GGT (Gamma Glutamyl Transferase/Transpeptidase) in their plasma and to analyse it statistically and there by evaluate the strength of the questionnaire.

\section{METHODS}

The study population includes two groups; group 1 consists of 50, self- reported, regular and excess alcohol drinking persons with AUDIT score less than 8; group 2 consists of persons with similar drinking behaviour with AUDIT score 8 and more irrespective of age. The purpose is to select an AUDIT score above which there is definite chance of alcoholic disorders by asking most of the questions to the respondents. Since an age old definite biomarker is used to assess the questionnaire a small group of respondents were selected with a scope to enlarge the study further in future.

\section{Exclusion Criteria}

1. Persons with liver disease.

2. Patients taking drugs like Phenytoin, Fibrates, Barbiturates, Rifampicin and Oestrogen-containing contraceptives.

3. Patients with carcinoma prostate. ${ }^{7}$

4. Patients who are not willing to give informed consent, however, are excluded from the study.

\section{Sample Collection and Analysis}

1. Random venous blood sample of $5 \mathrm{ml}$ was collected; the sample centrifuged; serum separated and analysed for the following;

2. Gamma glutamyltransferase (GGT) by method of Rosalki and Tarlo using spectrophotometer standardizing the PNA (Para Nitro Aniline)

3. Reference value $=$ less than $50 \mathrm{U} / \mathrm{L} 8$

\section{Statistics}

\section{Statistical Method}

Values are expressed as median, minimum and maximum. Comparisons were made with Mann-Whitney test when comparing two groups. Pairwise correlations were calculated with Pearson product-moment correlation coefficients, as required. A P-value $<0.05$ was considered statistically significant.

\section{RESULTS}

Table: GGT upper and lower limits, of Alcoholic and NonAlcoholic classified into different age cohorts, as indicated Values were given as median (Minimum - maximum); NA Not available

Mann Whitney test was used at 5\% level of significance.

\begin{tabular}{|c|c|c|c|c|c|c|c|}
\hline & \multicolumn{2}{|r|}{$\begin{array}{c}\text { Control } \\
\text { (Audit < } 8 \text { ) }\end{array}$} & \multicolumn{2}{|r|}{$\begin{array}{c}\text { Case } \\
\text { Audit } \geq 8 \text { ) }\end{array}$} & \multicolumn{2}{|r|}{ Overall } & \multirow[t]{2}{*}{$\begin{array}{c}\text { p- } \\
\text { value }\end{array}$} \\
\hline & $\mathbf{n}$ & $\begin{array}{c}\text { Median } \\
(\min -\max )\end{array}$ & $\mathbf{n}$ & $\begin{array}{c}\text { Median } \\
(\min -\text { max })\end{array}$ & $\mathbf{n}$ & $\begin{array}{c}\text { Median } \\
(\min -\max )\end{array}$ & \\
\hline \multicolumn{8}{|c|}{ Age in Years } \\
\hline$\leq 30$ & 22 & $\begin{array}{c}34.0 \\
(30.4-47.0) \\
\end{array}$ & 11 & $\begin{array}{c}40.0 \\
(33.0-46.0) \\
\end{array}$ & 33 & $\begin{array}{c}38.0 \\
(30.0-47.0) \\
\end{array}$ & 0.170 \\
\hline $30-45$ & 18 & $\begin{array}{c}41.5 \\
(31.0-92.0) \\
\end{array}$ & 14 & $\begin{array}{c}42.5 \\
(39.0-88.0)\end{array}$ & 32 & $\begin{array}{c}41.5 \\
(31.0-92.0) \\
\end{array}$ & 0.954 \\
\hline $45-55$ & 10 & $\begin{array}{c}81.0 \\
(33.0- \\
144.0) \\
\end{array}$ & 11 & $\begin{array}{c}92.0 \\
(84.0-141.0)\end{array}$ & 21 & $\begin{array}{c}92.0 \\
(33.0-144.0)\end{array}$ & 0.111 \\
\hline$>55$ & 0 & NA & 14 & $\begin{array}{c}107.0 \\
(92.0-172.0)\end{array}$ & 14 & $\begin{array}{c}107.0 \\
(92.0-172.0)\end{array}$ & NA \\
\hline Total & 50 & $\begin{array}{c}441.0 \\
(30.0-144.0) \\
\end{array}$ & 50 & $\begin{array}{c}85.5 \\
(33.0-172.0) \\
\end{array}$ & 100 & $\begin{array}{c}44.0 \\
(30.0-172.0) \\
\end{array}$ & $<0.001$ \\
\hline \multicolumn{8}{|c|}{ Table 1} \\
\hline
\end{tabular}

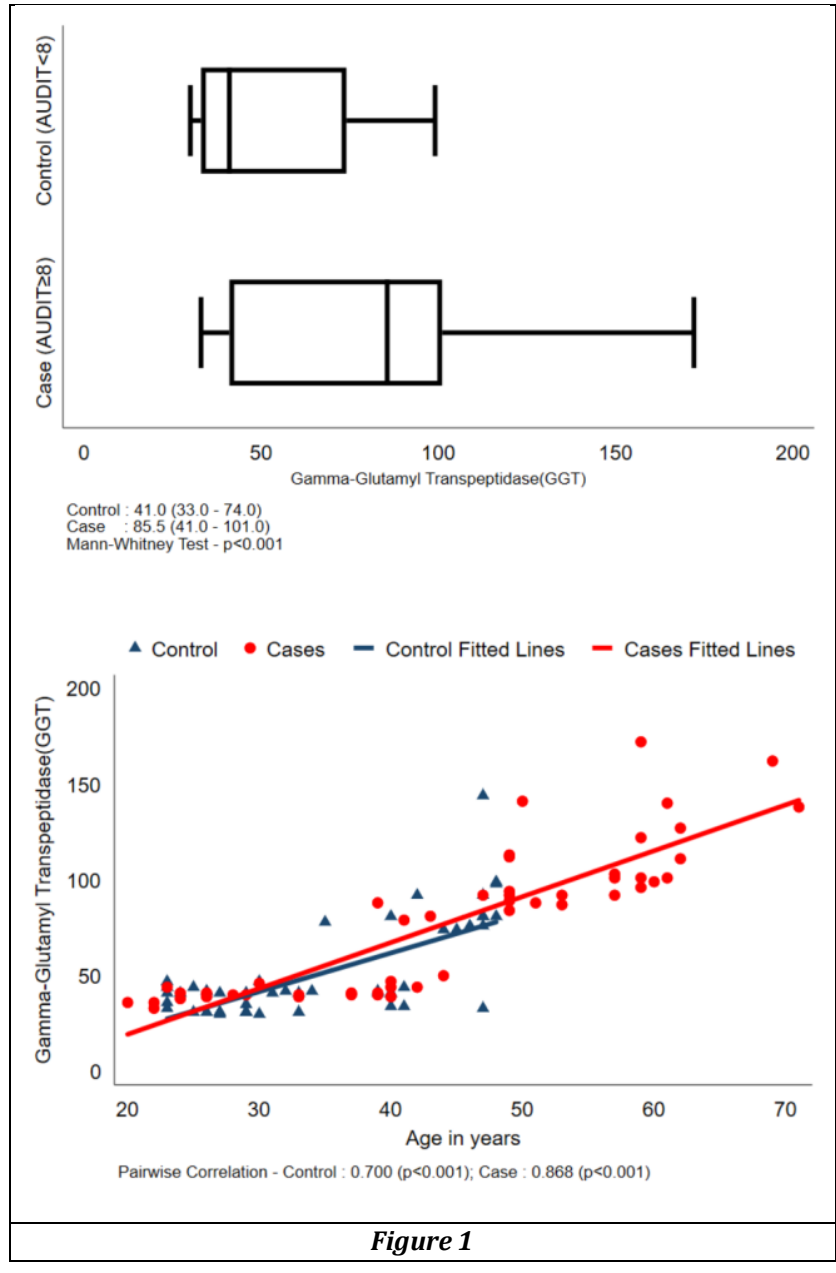

\section{DISCUSSION}

A cut of AUDIT score of 8 above which alcohol use disorders/alcoholism is usually expected, is selected and persons divided into two groups. Persons who consume alcohol but AUDIT score less than 8 and persons who consume alcohol with alcoholic questionnaire points of 8 and more than 8 are selected as mentioned above. In our study there was a steady increase of GGT as age goes up reveals that either there is increase in quantity or duration of drinking is 
evident and it starts increasing after 50 years of age. GGT doubles after 70 years of age but elevation in the control group has to be studied in detail with larger sample size and moreover enzyme levels in old age is usually at the upper limit of normal.

A population-based study by Kaur et al. in 11 villages of Tamil Nadu found the prevalence of alcohol consumption to be $69.8 \%$ among 497 men aged 25-64 years. ${ }^{8}$ A crosssectional study conducted in rural Goa by Dhupdale et al. ${ }^{9}$ among 410 subjects reported that the overall prevalence of alcohol consumption was $49 \%$ whereas the prevalence among males was $72 \%$. In both the study the AUDIT questionnaire was used. A study in rural Tamil Nadu among the general population found a prevalence of $9.4 \%$ whereas a study in rural Vellore by John et al found a prevalence of $24.6 \%$ among males and none among females. 10

In a similar study by using the AUDIT score cut off 8 by Verenkar et al in 2018 in Goa Medical College for estimating the prevalence, among the study participants was found to be $39.4 \%$ with total AUDIT score of eight or more was considered indicative of hazardous and harmful alcohol use as well as possible alcohol dependence. ${ }^{11} \mathrm{~A}$ study conducted by VV Ramanan et al in 2013 in Puducherry observe as per AUDIT score, $41.2 \%$ were at medium risk (AUDIT score 8-15) and $29.3 \%$ had high risk due to drinking (AUDIT score $\geq 16$ ) supports the view to use AUDIT score to categorize the risk of alcoholism. ${ }^{12}$ A similar situation would be expected in our state, more or less, as just a thin line separate Puducherry and Tamilnadu area and this might have been due to less price and easy availability of alcohol in Puducherry as well as in Tamilnadu.

As far as alcohol sale is concerned which is worrying in our state the total turnover is Rs 31,757 Crores per year ${ }^{13}$ an amount almost $1 / 5^{\text {th }}$ of the state's revenue. Is this same factor, someway or other stand in between prohibition and ill effects of alcoholism?

\section{CONCLUSIONS}

The present study supports the hypothesis that excessive alcohol drinking increases the plasma GGT. Self-reporting of alcohol drinking under-estimates the true risk. Thus, the use of GGT- the biological marker of alcohol drinking is a helpful tool for risk assessment. Excessive intake of alcohol, raised GGT level can go hand-in hand and a better understanding of the mechanism between alcohol drinking, biological markers, and the importance of AUDIT questionnaire is the base line tool for screening people of their drinking behaviour. Usually the AUDIT scoring method is followed in many studies to estimate the prevalence of drinking in a community. This study attempts to compare the questionnaire and the biological marker-GGT. The combination of questionnaire and the marker is equally as good as the questionnaire. The GGT tests the tissue levels while the questionnaire reveals the social behaviour of alcohol consumption. A larger study may throw light upon bias if it exists.

\section{REFERENCES}

[1] Park K. Stroke. In: Park K, edr. Parks Textbook of Social and Preventive Medicine. 16th edn. India: M/s Banarsidas Bhanot Publishers 2000: p. 280-1.

[2] Ray R, Mondal AB, Gupta K, et al. The Extent, Pattern and Trends of Drug Abuse in India: National Survey. New Delhi: United Nations Office on Drugs and Crimes and Ministry of Social Justice and Empowerment, Government of India, 2004.

[3] Singh Z. Alcohol or health: choice is yours. The Health Agenda 2014;2(4):108-11.

[4] Susan C. Enzymology. In: Anderson SC, Cockayne S, eds. Clinical chemistry - concepts and applications. $2^{\text {nd }}$ edn. Philadelphia: W.B. Saunders 1993: p. 268-9.

[5] Brown RL. Alcohol use disorders. In: Rakel RE, Bope ED, eds. Conn's Current Therapy 2007. Saunders 2007: p. 1273-5.

[6] Rosalki SB. Clinical biochemistry of alcohol. In: David L Williams DL, Marks V, eds. Scientific foundations of biochemistry in clinical practice. $2^{\text {nd }}$ edn. Butterworth Heinemann Publication 1994: p. 121, 128 \& 129.

[7] Ripple MO, Pickhardt PA, Wilding G, et al. Alteration in Gamma-glutamyl transpeptidase activity and messenger RNA of human prostate carcinoma cells by androgen. Cancer Research 1997;57(12):2428-33.

[8] Kaur P, Rao SR, Radhakrishnan E, et al. High prevalence of tobacco use, alcohol use and overweight in a rural population in Tamil Nadu, India. J Postgrad Med 2011;57(1):9-15.

[9] Dhupdale NY, Motghare DD, Ferreira AMA, et al. Prevalence and pattern of alcohol consumption in rural Goa. Indian J Commun Med 2006;31(2):104-5.

[10] Kumar SG, Premarajan KC, Subitha L, et al. Prevalence and pattern of alcohol consumption using Alcohol Use Disorders Identification Test (AUDIT) in Rural Tamil Nadu, India. J Clin Diagn Res 2013;7(8):1637-9.

[11] Verenkar YJ, Vaz FS. Prevalence and pattern of alcohol consumption using Alcohol Use Disorder Identification Test (AUDIT) among students at a medical college in Goa, India. Int J Community Med Public Health 2018;5(7):2935-8.

[12] Ramanan VV, Singh SK. A study on alcohol use and its related health and social problems in rural Puducherry, India. J Family Med Prim Care 2016;5(4):804-8.

[13] Md. Imranullah S. HC mulls over prosecuting state for alcohol-related crime. The Hindu, Dt: 28.3.19; 2019;142(72):2. 